\title{
Temporal analysis of an unprecedented data set for the $\gamma$-ray blazar 1ES 1215+303: \\ Fermi-LAT and VERITAS light curves spanning ten years
}

\author{
Janeth Valverde $^{* \dagger}$, Deirdre Horan ${ }^{\dagger}$, Giuliana Noto ${ }^{\ddagger}$, Reshmi Mukherjee ${ }^{\ddagger}$, Denis \\ Bernard ${ }^{\dagger}$ \\ ${ }^{\dagger}$ LLR/Ecole Polytechnique, Palaiseau, France. \\ ${ }^{\ddagger}$ Barnard College, Columbia University, New York, NY, United States. \\ E-mail: valverdedlir.in2p3.fr
}

on behalf of the Fermi-LAT \& VERITAS Collaborations

\begin{abstract}
We present here the results of the analysis of the $\gamma$-ray blazar, 1ES $1215+303$, over a 10 -year period, from 2008 to 2017, measured at high energies (HE; $200 \mathrm{MeV}<E<100 \mathrm{GeV}$ ) by the Fermi Large Area Telescope (LAT) and at very high energies (VHE; $E>100 \mathrm{GeV}$ ) by Fermi-LAT and VERITAS. This is the longest temporal study of this high-frequency-peaked BL Lac object (HBL) at $\gamma$-ray energies to date. The spectrum follows a log parabola over this time period, and its HE and VHE spectra are well-connected. Its flux is sufficiently strong at HE to allow us to bin the Fermi-LAT data in 3-day intervals, enabling us to investigate the temporal evolution of the flux in unprecedented detail. Several flaring episodes were detected and evidence for an overall trend of increasing flux over the span of the 10 years was observed. These light curves, in addition to the spectra, are presented. This unique data set will help us to advance our understanding of the underlying physical processes in blazar jets.
\end{abstract}

7th Fermi Symposium 2017

15-20 October 2017

Garmisch-Partenkirchen, Germany

\footnotetext{
* Speaker.
} 


\section{Introduction}

The largest population of sources detected by the current generation of space-based and groundbased $\gamma$-ray telescopes are blazars. They comprise more than $50 \%$ of the associated sources in the Third Fermi catalogue (3FGL) [1], more than 70\% of the Third Catalog of Hard Fermi-LAT Sources [2], and more than $30 \%$ of the objects detected at $\mathrm{TeV}$ energies ${ }^{1}$. Blazars are a subcategory of active galactic nuclei, very powerful systems believed to be powered by a central supermassive black hole, in which the relativistic jet is approximately coaligned with the line of sight of the Earth [3]. BL Lac objects are a further subclass of blazars, which usually do not have significant emission or absorption features in their optical spectra [4]. The study of high-flux variability of blazars allows us to put constraints on the size and location of the emitting region, while at the same time enabling us to probe the particle acceleration mechanisms in the blazar jets. Detailed studies across the entire $\gamma$-ray range allow the study the extragalactic background light (EBL, [5]) due to the TeV photon absorption by pair production, which can be used to estimate the density of the EBL [6].

1ES $1215+303$ (B2 1215+30, ON 325) is a blazar located at R. A. $(J 2000)=12^{h} 17^{m} 48.5^{s}$ and Dec. $(J 2000)=+30^{\circ} 07^{\prime} 00^{\prime \prime} 6$. It was first reported in the 1970 B2 $408 \mathrm{MHz}$ Bologna Northern Cross telescope radio catalogue [7] (hence the name B2), and has been observed at other wavelengths since then, including its first detection at VHE on 2011-01-[02-05] by the MAGIC collaboration (ATel \#3100, [8]). It has been classified as a high-frequency peaked [9] BL Lac [10] with a powerlaw spectrum, and its redshift was recently confirmed to be $z=0.131$, by optical spectroscopy [11]. There have been two separate detections of flaring activities from 1ES 1215+303 at VHE reported by VERITAS [12], and another two at HE, detected with the Fermi-LAT [13][12]. The first HE flare for this source occurred on 2008-10-[10-15] with a reported flux of $\mathrm{F}_{(E>300 \mathrm{MeV})} \sim$ $15 \times 10^{-8} \mathrm{~cm}^{-2} \mathrm{~s}^{-1}$ in a weekly light curve as part of a broader study of variability that included another 105 blazars [13]. The second HE flare occurred with a coincident counterpart at VHE on 2014-02-08 and was described in a detailed publication [12], which also reported the 2013-02-07 VHE flare. Moreover, 1ES 1215+303 was the subject of a long-term multi-wavelength study [14] that covered the time range from 2008 to 2012 with a $8.9 \sigma$ significance for energies greater than $100 \mathrm{GeV}$. In this contribution we report our preliminary results on the long-term variability and characteristics of 1ES $1215+303$ in its different states using data collected with the Fermi-LAT and VERITAS from 2008 to 2017.

\section{Instruments}

\subsection{VERITAS}

The Very Energetic Radiation Imaging Telescope Array System (VERITAS) is an array of four imaging atmospheric Cherenkov telescopes located at the Fred Lawrence Whipple Observatory in southern Arizona, U.S.A. ( $31^{\circ} 40^{\prime} \mathrm{N}, 110^{\circ} 57^{\prime} \mathrm{W}, 1.3 \mathrm{~km}$ a.s.1.). Each of the four telescopes comprises a 12-m diameter mirror, of the Davies Cotton design, and an imaging camera with 499 high-quantum-efficiency photomultiplier tubes. The four telescopes operate in stereo mode to cover a field of view of approximately $3.5^{\circ}$ with an angular resolution of $0.08^{\circ}$ at $1 \mathrm{TeV}(68 \%$ containment

\footnotetext{
${ }^{1}$ http: //tevcat2.uchicago.edu/
} 
radius). The energy range of VERITAS goes from $85 \mathrm{GeV}$ to above $30 \mathrm{TeV}$ (spectral reconstruction possible from $100 \mathrm{GeV}$ ) with an energy resolution of $15-25 \%$ and an effective collection area for a $1 \mathrm{TeV}$ photon on the order of $10^{5} \mathrm{~m}^{2}$. A source with a flux of $1 \%$ that of the Crab Nebula, the standard candle at these energies, is detectable at 5 standard deviations above background in approximately 25 hours [15]. The data were analysed using the two standard VERITAS analysis software packages [16], and produced results that were in excellent agreement with each other.

\subsection{Fermi-LAT}

The Large Area Telescope (LAT), on board the Fermi Gamma-ray Space Telescope, is a pairconversion detector that covers the energy range from $20 \mathrm{MeV}$ to more than $500 \mathrm{GeV}$ with a field of view of approximately $2.4 \mathrm{sr}$ [17]. The main observation mode of the Fermi-LAT is survey mode during which the LAT scans the entire sky every $3 \mathrm{hrs}$. We analyzed the Fermi-LAT data for 1ES 1215+303 from 2008-08-04, the start of the mission, up until to 2017-09-04. The data analysis was performed using the Fermi Science Tools package, version v10r0p5, and the latest P8R2_SOURCE_V6 instrument response functions. We have considered a maximum zenith angle of $90^{\circ}$ in order to reduce contributions from the Earth limb; and photons with energies greater than $100 \mathrm{MeV}$. For each data sample, we considered photons in a $10^{\circ}$ region of interest centered at the position of 1ES $1215+303$; and contributions from sources from the $3 \mathrm{FGL}$ within a $16.2^{\circ}$ region were included. None of the residual maps obtained in our analysis showed evidence for any additional excess. For the modeling of 1ES 1215+303, we considered both a power-law (PL) and a log-parabola (LP) spectral model (the second simplest model used in the 3FGL).
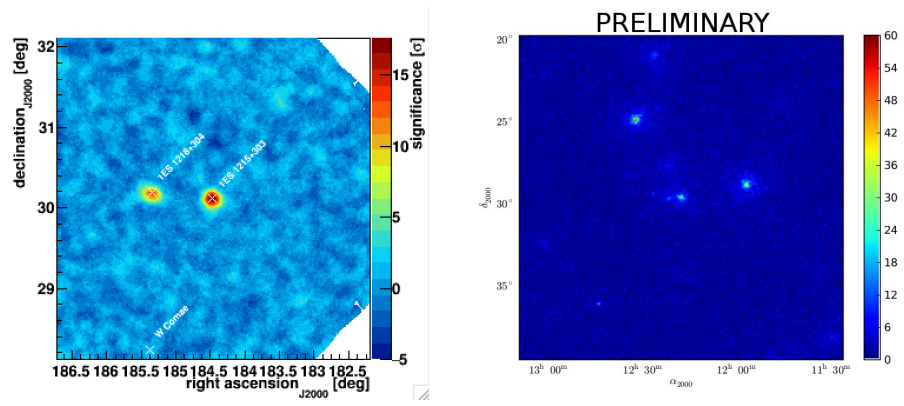

Figure 1: VERITAS (left) and LAT (right) 2015 1ES 1215+303 Skymaps.

\section{Preliminary results}

Fig. 2 shows the long-term light curves with VERITAS 1-day data points on the top and 3-day LAT data on the bottom. Zoomed-in image of 1-day light curves are shown for the brightest flares of 1ES $1215+303$ for the LAT data, and 5 min bins for the brightest VERITAS flare. The LAT upper limits are shown as green downward triangles. The VERITAS light curve includes archival data up to 2014; the 2015-2017 data were not previously published. An additional interesting feature can be observed in Fig. 3, where a possible increase in the average flux of 1ES 1215+303 is shown in 30-day and 10-day-binned light curves. A detailed analysis of the quiescent state will be necessary in order to understand this possible trend. Moreover, we report the detection of a coincidental GeVTeV flare on 2017-04-01. The SED of this flare can be seen in Fig. 4 together with the SED of the entire 2017-01-01 to 2017-06-30 season. The SED of the season without the two LAT-detected 
flaring epochs is also shown. In Fig. 4, the GeV-TeV data are observed to be well-connected in all of the SEDs. The EBL-absorbed [18] LAT spectral models were extrapolated to the VERITAS energy range. We observe that the LP SED is more compatible than the PL model with the TeV observations.

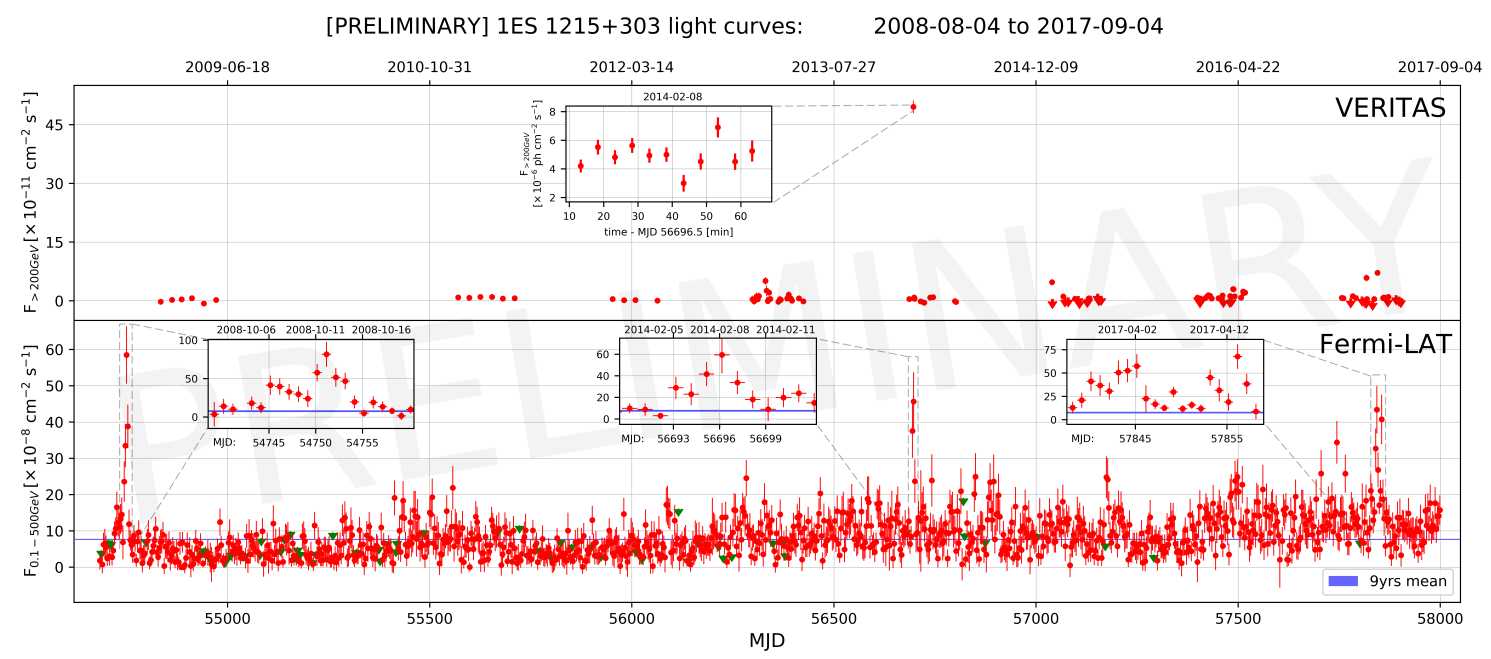

Figure 2: Long-term light curves: VERITAS 1-day data points on the top and 3-day LAT data on the bottom.

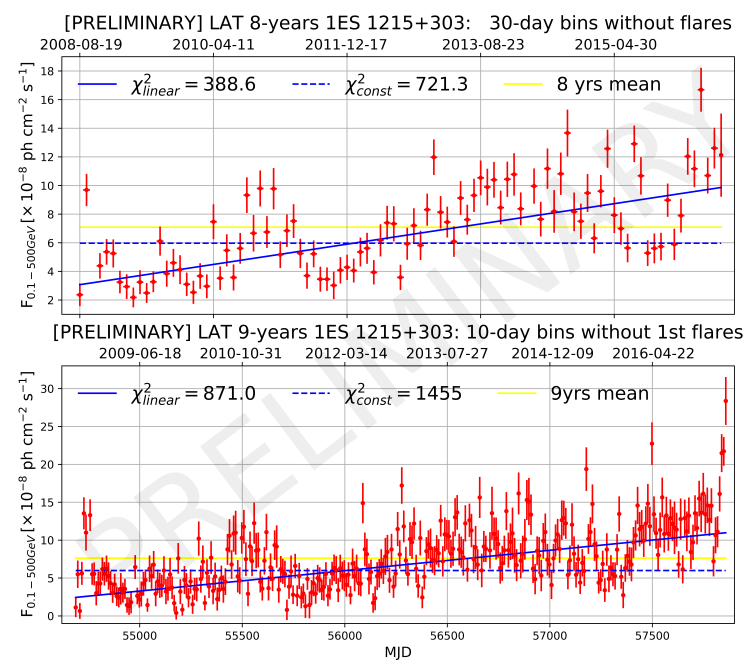

Figure 3: Top: 30-day binned light curve. Bottom: 10-day binned light curve. Both of them exclude the brightest flares. We observe a preference for a linear increase of the flux over a constant average in both data sets.
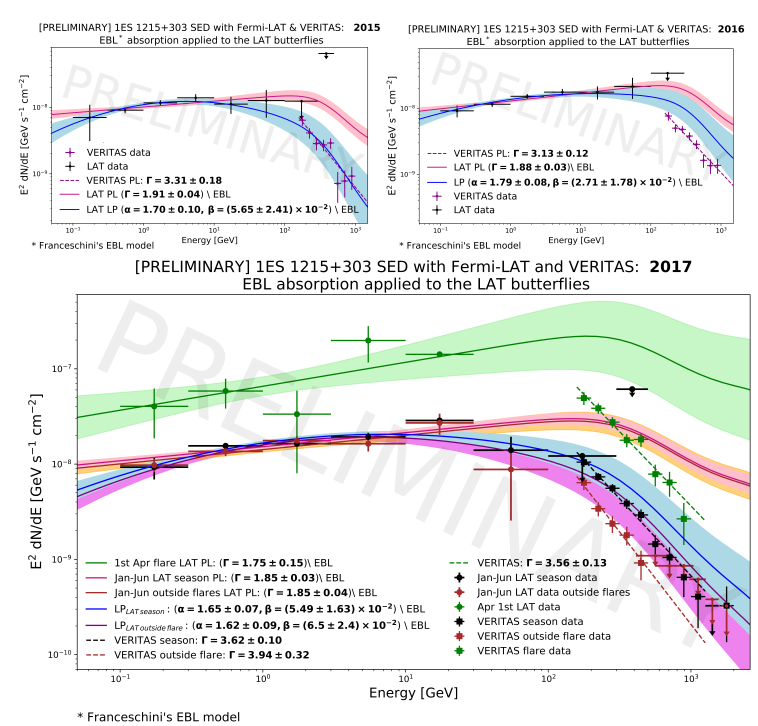

Figure 4: SEDs of years 2015 (top left), 2016 (top right) and 2017 (bottom). The flaring and quiescent states SEDs of year 2017 are additionally shown.

\section{Discussion}

The LAT data have demonstrated a preference for a log-parabola fit to the GeV spectrum (a power-law fit was rejected at the $5.26 \sigma$ level with the first 8 years of LAT data). This indicates that the $\gamma$-ray spectrum of this blazar begins to turn over in the LAT energy range, i.e., at energies below those that can be attributed purely to absorption by the EBL thus indicating curvature intrinsic to 
the source itself. The EBL-absorbed LAT log-parabola spectrum is consistent with the spectrum measured by VERITAS at higher energies, and therefore already subjected to the effects of EBL absorption. Unlike what has been observed for other blazars at $\mathrm{TeV}$ [19][20] and $\mathrm{GeV}$ energies [21], the spectral index of this blazar does not show significant evidence for hardening when it enters a higher flux state (Fig. 4). This relationship will be investigated further in our ongoing more-detailed analysis [22] as this relationship has been shown to be complex when shorter-term spectra were derived - e.g. for the case of Mrk 421 [23].

The fact that we were able to measure the GeV flux of this blazar in such unprecedented detail over a ten-year span has allowed us to evaluate the evolution of the flux with time. An interesting feature that we have already found is an apparent increasing trend in its flux over the duration of the observations (Fig. 3). We find that, for the $\mathrm{GeV} \gamma$-ray flux, an increasing-flux model is preferred to a constant flux at more than $15 \sigma$ level. Additionally, a preliminary yearly study of the complete LAT data set has shown some indication of a spectral-hardening trend of 1ES 1215+303 as a function of time. We are investigating this potential trend in the flux and spectral index, excluding the flaring epochs, and will present a more in-depth study in a subsequent publication that is in preparation between the Fermi-LAT and VERITAS Collaborations [22].

In conclusion, this unique data set will help us to advance our understanding of the underlying physical processes in blazar jets through the investigation of the variability, the search for periodicity and the cross-correlation studies between different wavelengths. Moreover, further investigation of the trend of long-term increasing flux and potential spectral hardening is ongoing.

Table 1: Summary of preliminary results ${ }^{a}$.

\begin{tabular}{ccccc}
\hline \hline \multirow{2}{*}{ Instrument } & Dates & Live time [min.] & Sign. & $\Gamma$ \\
\hline \hline \multirow{5}{*}{ VERITAS } & 2015-01-17 to 2015-05-19 & 979.4 & $17.1 \sigma$ & $-3.31 \pm 0.18$ \\
& 2016-01-12 to 2016-05-09 & 1324 & $26.4 \sigma$ & $-3.13 \pm 0.12$ \\
& 2017-01-03 to 2017-05-29 & 1473.95 & $33.0 \sigma$ & $-3.62 \pm 0.10$ \\
& $2015-01-17$ & 114.3 & $17.1 \sigma$ & $-2.96 \pm 0.18$ \\
& $2017-04-01$ & 150.1 & $28.9 \sigma$ & $-3.56 \pm 0.13$ \\
\hline \multirow{5}{*}{ Fermi-LAT } & & & & \\
& & & $37.4 \sigma$ & $-1.91 \pm 0.04$ \\
& $2015-01-01$ to $2015-06-30$ & & $54.4 \sigma$ & $-1.88 \pm 0.03$ \\
& 2016-01-01 to 2015-06-30 & & $139 \sigma$ & $-1.85 \pm 0.02$ \\
& 2017-01-01 to 2017-06-30 & & $11.2 \sigma$ & $-1.92 \pm 0.01$ \\
& 2008-08-04 to 2017-09-04 & & & \\
\hline \hline
\end{tabular}

${ }^{a}$ Refer to the paper in preparation for the final numbers.

See https: / /veritas.sao.arizona.edu for the VERITAS acknowledgements.

The Fermi-LAT Collaboration acknowledges support for LAT development, operation and data analysis from NASA and DOE (United States), CEA/Irfu and IN2P3/CNRS (France), ASI and INFN (Italy), MEXT, KEK, and JAXA (Japan), and the K.A. Wallenberg Foundation, the Swedish Research Council and the National Space Board (Sweden). Science analysis support in the operations phase from INAF (Italy) and CNES (France) is also gratefully acknowledged. This work performed in part under DOE Contract DE-AC02-76SF00515.

\section{References}

[1] F. Acero et al. 2015, Fermi Large Area Telescope Third Source Catalog, APJS 218, 23.

[2] M. Ajello et al. 2017, 3FHL: The Third Catalog of Hard Fermi-LAT Sources, APJS 232, 18, (3FHL).

[3] V. Beckmann \& C. Shrader 2012, The AGN phenomenon: open issues, in proceedings of the 9th INTEGRAL Workshop, POS (INTEGRAL 2012) 069.

[4] C. D. Dermer \& B. Giebels 2016, Active galactic nuclei at gamma-ray energies, Comptes Rendus Physique 17, 594. 
[5] M. G. Hauser \& E. Dwek 2001, The Cosmic Infrared Background: Measurements and Implications, ARAA 39, 249.

[6] M. Ackermann et al. 2012, The Imprint of the Extragalactic Background Light in the Gamma-Ray Spectra of Blazars, Science 338, 1190.

[7] G. Colla et al. 1970, A catalogue of 3235 radio sources at $408 \mathrm{MHz}, \mathrm{A} \& A S \mathbf{1}, 281$.

[8] J. Aleksić et al. 2012, Discovery of VHE $\gamma$-rays from the blazar 1ES 1215+303 with the MAGIC telescopes and simultaneous multi-wavelength observations, A\&A 544, A 142.

[9] M. Ackermann et al. 2011, The Second Catalog of Active Galactic Nuclei Detected by the Fermi Large Area Telescope, ApJ 743, 171.

[10] I. W. A. Browne et al. 1971, Two Bright New Quasi-stellar Radio Sources, Nature 231, 515.

[11] S. Paiano et al. 2017, On the Redshift of TeV BL Lac Objects, ApJ 837, 144.

[12] A. U. Abeysekara et al. 2017, A Luminous and Isolated Gamma-Ray Flare from the Blazar B2 $1215+30$, ApJ 836, 205.

[13] A. A. Abdo et al. 2010, Gamma-ray Light Curves and Variability of Bright Fermi-detected Blazars, ApJ 722, 520.

[14] E. Aliu et al. 2013, Long Term Observations of B2 1215+30 with VERITAS, ApJ 779, 92.

[15] J. Holder et al. 2011, VERITAS: Status and Highlights, in 32nd ICRC, 12, 137 [arXiv:1111.1225].

[16] V. A. Acciari et al. 2008, VERITAS Observations of the $\gamma$-Ray Binary LS I +61 303, ApJ 679, 1427.

[17] W. B. Atwood et al. 2009, The Large Area Telescope on the Fermi Gamma-Ray Space Telescope Mission, ApJ 697, 1071.

[18] A. Franceschini, G. Rodighiero \& M. Vaccari 2008, Extragalactic optical-infrared background radiation, its time evolution and the cosmic photon-photon opacity, A\&A 487, 837.

[19] A. Djannati-Atai et al. 1999, Very High Energy Gamma-ray spectral properties of MKN 501 from CAT Cerenkov telescope observations in 1997, A\&A 350, 17.

[20] V. A. Acciari et al. 2011, Multiwavelength Observations of the Very High Energy Blazar IES $2344+514$, ApJ 738,169.

[21] Y. T. Tanaka et al. 2016, A significant hardening and rising shape detected in the MeV/GeV $v F_{v}$ spectrum from the recently discovered very-high-energy blazar $S 40954+65$ during the bright optical flare in 2015 February, PASJ 68, 51.

[22] Fermi-LAT \& VERITAS Collaborations, ApJ, in preparation.

[23] A. U. Abeysekara et al 2017, A Search for Spectral Hysteresis and Energy-dependent Time Lags from X-Ray and TeV Gamma-Ray Observations of Mrk 421, ApJ 834, 2. 